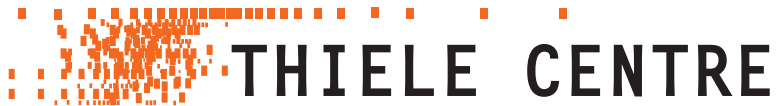 FOR APPLIED MATHEMATICS IN NATURAL SCIENCE
}

Time Change, Volatility, and Turbulence

Ole E. Barndorff-Nielsen and Jürgen Schmiegel 



\section{Time Change, Volatility, and Turbulence}

This Thiele Research Report is also Research Report number 499 in the Stochastics Series at Department of Mathematical Sciences, University of Aarhus, Denmark. 



\title{
Time Change, Volatility, and Turbulence
}

\author{
Ole E. Barndorff-Nielsen and Jürgen Schmiegel \\ Thiele Centre for Applied Mathematics in Natural Science, \\ Department of Mathematical Sciences, University of Aarhus, Denmark
}

July 30, 2007

\begin{abstract}
A concept of Volatility Modulated Volterra Processes is introduced. Apart from some brief discussion of generalities, the paper focusses on the special case of backward moving average processes driven by Brownian motion. In this framework, a review is given of some recent modelling of turbulent velocities and associated questions of time change and universality. A discussion of similarities and differences to the dynamics of financial price processes is included.
\end{abstract}




\section{Contents}

1 Introduction 3

2 Background $\quad 3$

2.1 Turbulence. . . . . . . . . . . . . . . . . . . . 4

2.2 Stylised features of finance and turbulence . . . . . . . . . . . . 4

2.3 Normal inverse Gaussian distributions . . . . . . . . . . . . . . 5

3 Volatility modulated Volterra processes 5

3.1 Volterra type processes . . . . . . . . . . . . . . 5

3.2 Volatility modulated Volterra processes . . . . . . . . . . . . . 7

3.3 Time change and VMVP . . . . . . . . . . . . . . 8

4 Time change in stationary processes 9

5 Universality in Turbulence $\quad \mathbf{1 0}$

5.1 Empirics . . . . . . . . . . . . . . . . . . . 10

5.2 Theoretical considerations . . . . . . . . . . . . . . 11

6 Modelling frameworks in finance and turbulence $\quad 12$

6.1 Finance . . . . . . . . . . . . . . . . . . . . 12

6.2 Turbulence. . . . . . . . . . . . . . . . . . . . 12

$\begin{array}{lll}7 & \text { Increment processes } & 13\end{array}$

8 Time change in finance and turbulence $\quad \mathbf{1 5}$

8.1 Finance . . . . . . . . . . . . . . . . . . . 15

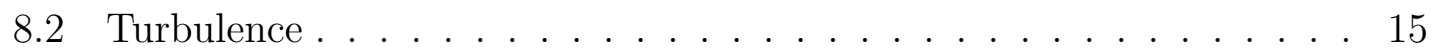

9 Universality: Modelling 16

9.1 Primitive Universality Model . . . . . . . . . . . . . . . . . . 16

9.2 Refined Universality Model . . . . . . . . . . . . . . . . 16

10 Concluding remarks $\quad 17$

$\begin{array}{ll}\text { A The Normal inverse Gaussian Law } & 17\end{array}$ 


\section{Introduction}

Change of time is an important concept in stochastic analysis and some of its applications, especially in mathematical finance and financial econometrics, with quadratic variation and its interpretation as integrated squared volatility playing a key role. (A rather comprehensive treatment of this will be available in [BNShi08].) On the other hand there are well known similarities, as well as important differences, between the dynamics of financial markets and of turbulence. From these prospects, the present paper discusses some recent modelling in turbulence and associated questions of time change.

To set the discussion in perspective, a general concept of Volatility Modulated Volterra Processses is introduced. This would seem to be of some rather wide interest in mathematical modelling. Here we focus on its relevance for stochastic modelling of turbulence. For a masterly overview of the main approaches to modelling of turbulence see [Shi07], cf. also [Shi06].

A summary comparison of main stylised features in finance and turbulence is given in the next Section. Of central importance is the fact that volatility is a key concept in turbulence as well as in finance, though in turbulence the phenomenon is referred to as intermittency.

The notion of change of time in mathematical finance and financial econometrics refers to an increasing stochastic process as the time change while in turbulence we have in mind a deterministic time change. We provide empirical and theoretical evidence for the existence of an affine deterministic time change in turbulence in terms of which the main component of the velocity vector in a turbulent flow behaves in a universal way over a wide range of scales. We also discuss the limitations of this type of universality and briefly outline the extension to a non-affine deterministic change of time and its relevance for universality of velocity increments.

Section 2 provides some background on turbulence and the similarities and differences between turbulence and finance, and recalls some features of the Normal inverse Gaussian distribution. Section 3 discusses volatility modulated Volterra processes and their behaviour under change of time. A more general discussion of change of time for stationary processes is presented in Section 4. Section 5 provides empirical and theoretical evidence for the relevance of change of time in turbulence. The potential of Volterra processes for modelling velocity fields in turbulence is outlined in Sections 6 and 7. Section 8 relates the concept of change of time to the particular setting of the proposed modelling frameworks in finance and turbulence. This leads to a primitive and a refined universality statement for turbulence in Section 9. Section 10 concludes.

\section{Background}

The statistics of turbulent flows and financial markets share a number of stylized features ([Gha96], [Pei04], [BNShi08] and [BN98a]). The counterpart of the velocity in turbulence is the log price in finance, and velocity increments correspond to log returns. The equivalent of the intermittency of the energy dissipation in turbulence is the strong variability of the volatility in financial markets. Subsection 2.1 briefly 
summarizes some basic information on turbulence, and subsection 2.2 lists the most important similarities and differences between turbulence and finance. The normal inverse Gaussian laws constitute a useful tool in both fields and some of the properties of these laws are recalled in the Appendix.

\section{$2.1 \quad$ Turbulence}

There is no generally accepted definition of what should be called a turbulent flow. Turbulent flows are characterized by low momentum diffusion, high momentum convection, and rapid variation of pressure and velocity in space and time. Flow that is not turbulent is called laminar flow. The non-dimensional Reynolds number $R$ characterizes whether flow conditions lead to laminar or turbulent flow. Increasing the Reynolds number increases the turbulent character and the limit of infinite Reynolds number is called the fully developed turbulent state.

The most prominent observable in a turbulent flow is the main component of the velocity field $V_{t}$ as a function of time $t$ and at a fixed position in space. A derived quantity is the temporal surrogate energy dissipation process describing the loss of kinetic energy due to friction forces characterized by the viscosity $\nu$

$$
\varepsilon_{t} \equiv \frac{15 \nu}{\bar{V}^{2}}\left(\frac{\mathrm{d} V_{t}}{\mathrm{~d} t}\right)^{2}
$$

where $\bar{V}$ denotes the mean velocity.

The temporal surrogate energy dissipation process takes into account the experimental condition where only a time series of the main component of the velocity vector is accessible. The temporal surrogate energy dissipation is a substitute for the true energy dissipation process (involving the spatial derivatives of all velocity components) for flows which are stationary, homogeneous and isotropic [Els96]. In the sequel we call such flows free turbulent flows. We refer to the temporal surrogate energy dissipation as the energy dissipation unless otherwise stated.

Since the pioneering work of Kolmogorov [Kol62] and Obukhov [Obu62], intermittency of the turbulent velocity field is of major interest in turbulence research. From a probabilistic point of view, intermittency refers, in particular, to the increase in the non-Gaussian behaviour of the probability density function (pdf) of velocity increments

$$
\Delta u_{s}=V_{t+s}-V_{t}
$$

with decreasing time scale $s$. Here we adopt the notation $\Delta u$ for velocity increments which is traditional in the turbulence literature. A typical scenario is characterized by an approximate Gaussian shape for the large scales, turning to exponential tails for the intermediate scales and stretched exponential tails for dissipation scales ([Cas90] and [Vin91], see also Figure 1).

\subsection{Stylised features of finance and turbulence}

The most important similarities between financial markets and turbulent flows are semiheavy tails for the distributions of log returns/velocity increments, the evolution of the densities of log returns/velocity increments across time scales with the 
heaviness of the tails decreasing as the time lag increases, and long range dependence of $\log$ returns/velocity increments. It is important to note that in spite of the long range dependence the autocorrelation of the log price process is essentially zero whereas the velocity field shows algebraic decay of the autocorrelation function. Other important differences are the skewness of the densities of velocity increments in contrast to the symmetry of the distribution of log returns in FX markets ${ }^{1}$ and the different behaviour of bipower variation [BNS04; BNSchS07]. Table 1 gives an overview of the differences and similarities between turbulence and finance.

\subsection{Normal inverse Gaussian distributions}

Intermittency/volatility is related to the heaviness of the tails and the non-Gaussianity of the distribution of velocity increments and log returns. In this respect, Normal inverse Gaussian (NIG) distributions are a suitable class of probability distributions which fit the empirical densities in both systems to high accuracy ([BN95; BN97], [RYD97], [FOR02], [BNBSch04], [BNSch06b]).

Figure 1 shows, as an example, the log densities of velocity increments $\Delta u_{s}$ measured in the atmospheric boundary layer for various time scales $s$. The solid lines denote the approximation of these densities within the class of NIG distributions. NIG distributions fit the empirical densities equally well for all time scales $s$.

A subsequent analysis of the observed parameters of the NIG distributions from many, widely different data sets with Reynolds numbers ranging from $R_{\lambda}=80$ up to $R_{\lambda}=17000$ (where $R_{\lambda}$ is the Taylor based Reynolds number) led to the formulation of a key universality law ([BNBSch04] and [BNSch07b]): The temporal development of a turbulent velocity field has an intrinsic clock which depends on the experimental conditions but in terms of which the one-dimensional marginal distributions of the normalized velocity differences become independent of the experimental conditions. Figure 2 provides an empirical validation of this.

\section{Volatility modulated Volterra processes}

This Section is divided into three subsections. The first briefly discusses Volterra type processes, the second introduces the concept of volatility modulated Volterra processes, and the third considers the behaviour of such processes under a change of time.

\subsection{Volterra type processes}

In this paper we shall be referring to processes of the form

$$
Y_{t}=\int_{-\infty}^{\infty} K(t, s) \mathrm{d} B_{s}+\chi \int_{-\infty}^{\infty} Q(t, s) \mathrm{d} s
$$

\footnotetext{
${ }^{1}$ For stocks, skewness of the distribution of log returns is observed. There, leverage is believed to be a key mechanism.
} 
as Browninan Volterra processes (BVP). Here $K$ and $Q$ are deterministic functions, sufficiently regular to give suitable meaning to the integrals; Furthermore, $\chi$ is a constant and $B$ denotes standard Brownian motion.

Example 1 (Fractional Brownian motion). As is well known (cf, for instance [SaTa94]) fractional Brownian motion can be written as

$$
B_{t}^{H}=\int_{-\infty}^{\infty}\left[(t-s)_{+}^{H-1 / 2}-(-s)_{+}^{H-1 / 2}\right] \mathrm{d} B_{s}
$$

Of particular interest are the backward Volterra processes, i.e. where $K(t, s)$ and $Q(t, s)$ are 0 for $s>t$. In this case formula (2) takes the form

$$
Y_{t}=\int_{-\infty}^{t} K(t, s) \mathrm{d} B_{s}+\chi \int_{-\infty}^{t} Q(t, s) \mathrm{d} s .
$$

Example 2. Fractional Brownian motion For $B$ a Brownian motion, the fractional Brownian motion with index $H \in(0,1)$ may alternatively, see [NoVaVir99], be represented as

$$
B_{t}^{H}=\int_{0}^{t} K(t, s) \mathrm{d} B_{s}
$$

where

$$
K(t, s)=c_{H}\left\{\left(\frac{t}{s}\right)(t-s)^{H-1 / 2}-\left(H-\frac{1}{2}\right) s^{1 / 2-H} \int_{s}^{t} u^{H-3 / 2}(u-s)^{H-1 / 2} \mathrm{~d} u\right\}
$$

and

$$
c_{H}=\left(\frac{2 \Gamma\left(\frac{3}{2}-H\right)}{\Gamma\left(H+\frac{1}{2}\right) \Gamma(2-2 H)}\right)^{1 / 2} .
$$

A more general type of Volterra processes are the Lévy Volterra processes (LVP), which are of the form

$$
Y_{t}=\int_{-\infty}^{\infty} K(t, s) \mathrm{d} L_{s}+\chi \int_{-\infty}^{\infty} Q(t, s) \mathrm{d} s
$$

where $L$ denotes a Lévy process on $\mathbb{R}$ and $K$ and $Q$ are deterministic kernels, satisfying certain regularity conditions.

Example 3. Fractional Lévy motion [Mar06] introduces Fractional Lévy motion $L^{H}$ for $H \in\left(\frac{1}{2}, 1\right)$ by the formula

$$
L_{t}^{H}=\int_{-\infty}^{\infty}\left[(t-s)_{+}^{H-1 / 2}-(-s)_{+}^{H-1 / 2}\right] \mathrm{d} L_{s}
$$

where $L$ is a Lévy process.

Stochastic integration in these general settings is discussed for BVP in [Hu03], [Dec05], [DecSa06], cf. also [NoVaVir99], and for LVP in [BeMar07].

For Brownian Volterra processes we shall refer to the following three conditions: For all $s, t \in \mathbb{R}$ 
C1

$$
K(t, \cdot) \in L^{2}(\mathbb{R}) \quad \text { and } \quad Q(t, \cdot) \in L^{2}(\mathbb{R})
$$

C2

$$
\begin{array}{lll}
K(s, s)=K_{0}>0 & \text { and } & K(t, s)=0 \text { for } s>t \\
Q(s, s)=Q_{0}>0 & \text { and } & Q(t, s)=0 \text { for } s>t
\end{array}
$$

C3 $K$ and $Q$ are differentiable with respect to their first arguments and, denoting the derivatives by $\dot{K}$ and $\dot{Q}$, we have

$$
\dot{K}(t, \cdot) \in L^{2}(\mathbb{R}) \text { and } \dot{Q}(t, \cdot) \in L^{2}(\mathbb{R}) .
$$

Under these conditions the covariance function of (3) exists and is, for $s \leq t$, given by

$$
R(s, t)=\operatorname{Cov}\left\{Y_{s}, Y_{t}\right\}=\int_{-\infty}^{s} K(t, u) K(s, u) \mathrm{d} u
$$

and the autocorrelation function may be written as

$$
r(s, t)=\int_{-\infty}^{s} \bar{K}(t, u) \bar{K}(s, u) \mathrm{d} u
$$

where

$$
\bar{K}(t, u)=K(t, u) /\|K(t, \cdot)\|
$$

and \|\| denotes the $L^{2}$ norm.

\subsection{Volatility modulated Volterra processes}

For modelling purposes it is of interest to consider Volatility modulated Volterra processes (VMVP) which we define (backward case) by

$$
Y_{t}=\int_{-\infty}^{t} K(t, s) \sigma_{s} \mathrm{~d} B_{s}+\chi \int_{-\infty}^{t} Q(t, s) \sigma_{s}^{2} \mathrm{~d} s
$$

where $\sigma>0$ is a stationary cadlag process on $\mathbb{R}$, embodying the volatility/intermittency.

On the further assumptions that the deterministic kernels $K$ and $Q$ satisfy conditions $\mathbf{C 1}-\mathbf{C} 3$, we have that $Y$ is a semimartingale, satisfying the stochastic differential equation

$$
\mathrm{d} Y_{t}=K_{0} \sigma_{t} \mathrm{~d} B_{t}+\chi Q_{0} \sigma_{t}^{2} \mathrm{~d} t+\int_{-\infty}^{t} \dot{K}(t, s) \sigma_{s} \mathrm{~d} B_{s}+\chi \int_{-\infty}^{t} \dot{Q}(t, s) \sigma_{s}^{2} \mathrm{~d} s .
$$

The quadratic variation of $Y$ is then, for $t \geq 0$,

$$
[Y]_{t}=K_{0}^{2} \tau_{t}
$$

where

$$
\tau_{t}=\int_{0}^{t} \sigma_{s}^{2} \mathrm{~d} s
$$


is the integrated squared volatility process. For $t<0$ we define $[Y]_{t}$ and $\tau_{t}$ by the same formulae (6) and (7). Then $[Y]$ is a continuous increasing stochastic process with $[Y]_{0}=0$.

Finally, we introduce the inverse process $\theta$ of $\tau$ by

$$
\theta_{t}=\inf \left\{s: \tau_{s} \geq t\right\} \text {. }
$$

\subsection{Time change and VMVP}

We say that a process $T$ on $\mathbb{R}$ is a time change provided $T$ is increasing with $T_{0}=0$. The time changes on $\mathbb{R}$ we shall be considering are in fact continuous and strictly increasing, with $T \rightarrow \pm \infty$ as $t \rightarrow \pm \infty$. (This is the case in particular for the processes $\tau$ and $\theta$ defined above.)

For $T$ a time change process on $\mathbb{R}$ and given a Volterra kernel $K$ we define a new Volterra kernel $K \circ T$ by

$$
K \circ T(t, s)=K\left(T_{t}, T_{s}\right) .
$$

Taking $T=\theta$ as given by (8) we may now rewrite $Y$ of ( 5$)$ as

$$
\begin{aligned}
Y_{t} & =\int_{-\infty}^{t} K(t, s) \mathrm{d} B_{\tau_{s}}+\chi \int_{-\infty}^{t} Q(t, s) \mathrm{d} \tau_{s} \\
& =\int_{-\infty}^{\tau_{t}} K\left(t, \theta_{u}\right) \mathrm{d} B_{u}+\chi \int_{-\infty}^{\tau_{t}} Q\left(t, \theta_{u}\right) \mathrm{d} u
\end{aligned}
$$

implying

$$
Y_{\theta_{t}}=\int_{-\infty}^{t} K \circ \theta(t, s) \mathrm{d} B_{s}+\chi \int_{-\infty}^{t} Q \circ \theta(t, s) \mathrm{d} s .
$$

In particular, if the volatility process $\sigma$ is independent of $B$ then, conditional on $[Y]$, $Y_{\theta}$ is a Volterra process with kernels $(K \circ \theta, Q \circ \theta)$ and the same driving Brownian motion as the VMVP $Y$.

Later in this paper we shall, in the context of turbulence, be concerned with affine time changes.

Remark 4. Affine time change Suppose $T_{t}=c t+c_{0}$ for some $c>0$ and a constant $c_{0}$. Applying this to (5) gives

$$
\begin{aligned}
Y_{c t+c_{0}} & =\int_{-\infty}^{c t+c_{0}} K\left(c t+c_{0}, s\right) \sigma_{s} \mathrm{~d} B_{s}+\chi \int_{-\infty}^{c t+c_{0}} Q\left(c t+c_{0}, s\right) \sigma_{s}^{2} \mathrm{~d} s \\
& =\int_{-\infty}^{t} K\left(c t+c_{0}, c u+c_{0}\right) \sigma_{c u+c_{0}} \mathrm{~d} B_{c u+c_{0}}+c \chi \int_{-\infty}^{t} Q\left(c t+c_{0}, c u+c_{0}\right) \sigma_{c u+c_{0}}^{2} \mathrm{~d} u
\end{aligned}
$$

i.e.

$$
Y_{c t+c_{0}}=\int_{-\infty}^{t} K_{c, c_{0}}(t, s) \sigma_{c s+c_{0}} \mathrm{~d} \tilde{B}_{s}+\chi \int_{-\infty}^{t} Q_{c, c_{0}}(t, s) \sigma_{c s+c_{0}}^{2} \mathrm{~d} s
$$

with $K_{c, c_{0}}(t, s)=\sqrt{c} K\left(c t+c_{0}, c s+c_{0}\right), Q_{c, c_{0}}(t, s)=c Q\left(c t+c_{0}, c s+c_{0}\right)$ and where $\tilde{B}_{s}=c^{-1 / 2} B_{c s}$ is a Brownian motion. Thus the transformed process follows again a VMVP but now with volatility process $\sigma_{c \cdot+c_{0}}$ (and kernels $K_{c, c_{0}}$ and $Q_{c, c_{0}}$ ). 


\section{Time change in stationary processes}

Let $Y$ and $Y^{*}$ be stationary stochastic processes on $\mathbb{R}$ and let $X$ and $X^{*}$ be the corresponding increment processes given by $X_{t}=Y_{t}-Y_{0}$ and $X_{t}^{*}=Y_{t}^{*}-Y_{0}^{*}$. The present Section discusses distributional relations between $X$ and $X^{*}$ under various assumptions. Note first, however, that only affine time changes preserve stationarity in a stationary process.

Assuming $\operatorname{Var}\left\{Y_{t}\right\}=\operatorname{Var}\left\{Y_{t}^{*}\right\}=\omega^{2}$, say, and denoting the autocorrelation functions of $Y$ and $Y^{*}$ respectively by $r$ and $r^{*}$ we have

$$
\operatorname{Var}\left\{X_{t}\right\}=2 \omega^{2} \bar{r}(t) \quad \text { and } \operatorname{Var}\left\{X_{t}^{*}\right\}=2 \omega^{2} \bar{r}^{*}(t)
$$

where $\bar{r}(t)=1-r(t)$ and $\bar{r}^{*}(t)=1-r^{*}(t)$.

Let $\psi(t)$ be a a time change, and suppose that

$$
X_{t} \stackrel{\text { law }}{=} X_{\psi(t)}^{*} \quad \text { for every } t \in \mathbb{R} .
$$

As discussed in Section 2.3 this type of behaviour has been found in free turbulence.

Assumption (13) and the relation (12) imply

$$
r(t)=r^{*}(\psi(t)),
$$

and provided both $r$ and $r^{*}$ are strictly decreasing and continuous functions we have that the time change $\psi$ is expressible as

$$
\psi(t)=\rho^{*}(r(t)),
$$

$\rho^{*}$ denoting the inverse function of $r^{*}$

In case the statistical analysis of observations from $X$ and $X^{*}$ has shown good agreement with the Ansatz (13), it is then natural to ask whether (13) is simply a reflection of the more sweeping Ansatz

$$
X \stackrel{\text { law }}{=} X_{\psi(\cdot)}^{*},
$$

saying that $X$ and $X^{*}$ are equal in law as processes and not just pointwise as in (13). This Ansatz implies, in particular, that $\psi$ must be affine since, as mentioned earlier, only affine time changes preserve stationarity.

In fact, the weaker assumption of second order agreement of $X$ and $X^{*}$ already implies that $\psi$ is affine, as we show now.

Suppose that for all $0 \leq s \leq t$

$$
\begin{gathered}
\mathrm{E}\left\{X_{\psi(t)}^{* 2}\right\}=\mathrm{E}\left\{X_{t}^{2}\right\} \\
\mathrm{E}\left\{X_{\psi(s)}^{*} X_{\psi(t)}^{*}\right\}=\mathrm{E}\left\{X_{s} X_{t}\right\} .
\end{gathered}
$$

(Note that since we have assumed that $Y$ and $Y^{*}$ are stationary, necessarily $\mathrm{E}\left\{X_{t}\right\}=$ $0=\mathrm{E}\left\{X_{\psi(t)}^{*}\right\}$.) We will then have

$$
\mathrm{E}\left\{\left(X_{\psi(t)}^{*}-X_{\psi(s)}^{*}\right)^{2}\right\}=\mathrm{E}\left\{\left(X_{t}-X_{s}\right)^{2}\right\}
$$


Further, by (12), on the one hand

$$
\mathrm{E}\left\{\left(X_{\psi(t)}^{*}-X_{\psi(s)}^{*}\right)^{2}\right\}=\mathrm{E}\left\{\left(Y_{\psi(t)}^{*}-Y_{\psi(s)}^{*}\right)^{2}\right\}=2 \omega^{2} \bar{r}^{*}(\psi(t)-\psi(s))
$$

while on the other

$$
\mathrm{E}\left\{\left(X_{t}-X_{s}\right)^{2}\right\}=\mathrm{E}\left\{\left(Y_{t}-Y_{s}\right)^{2}\right\}=2 \omega^{2} \bar{r}(t-s) .
$$

This implies

$$
\bar{r}^{*}(\psi(t)-\psi(s))=\bar{r}(t-s)
$$

or, equivalently, by (14),

$$
\psi(t)-\psi(s)=\psi(t-s)
$$

which can only hold for $\psi$ affine,

$$
\psi(t)=c t+c_{0}
$$

for some $c>0$ and a constant $c_{0}$.

\section{$5 \quad$ Universality in Turbulence}

In this Section we discuss the empirical support for the existence of affine, intrinsic (one for each experiment), time changes such that for a wide range of time scales the densities of turbulent velocity increments obtained from different experiments collapse. This leads to the formulation of a primitive universality model for turbulence (see subection 9.1). At very small or very large time scales, deviations from affinity are observed. This then leads to the formulation of a refined universality model (see subsection 9.2). The dynamical aspects of this refined universality model are briefly discussed in the concluding Section 10.

In comparing the equivalence under time change to existing theory of turbulence and to empirical evidence it is illuminating to relate the discussion to the well established fact (cf. Section 2.3) that in free turbulence the distributions of velocity differences over fixed time spans are closely describable by the NIG law.

\subsection{Empirics}

The statistical analysis of a large number of different turbulent data sets revealed the existence of a type of universality which states that the densities of velocity increments are well described within the class of NIG distributions and, moreover, the densities of the increments of the normalized velocity field obtained from different experiments collapse as long as the intrinsic time scales are measured in terms of the scale parameter of the approximate NIG distributions. Here, the velocity field is normalized by its standard deviation. We denote the normalized velocity component by $\tilde{V}=V / \sqrt{\operatorname{Var}(V)}$. Then we have the empirical result, denoting the corresponding velocity increments by $\Delta \tilde{u}$,

$$
\Delta \tilde{u}_{t} \stackrel{\text { law }}{=} \Delta \tilde{u}_{\psi(t)}^{*}
$$

where

$$
\psi(t)=\overleftarrow{\delta}^{*}(\delta(t))
$$


and where $\delta(t)$ and $\delta^{*}(t)$ are the scale parameters of the approximate NIG distributions of $\Delta \tilde{u}_{t}$ and $\Delta \tilde{u}_{t}^{*}$, respectively. Here $\Delta \tilde{u}_{t}$ refers to the velocity increments for a given turbulent experiment and the superscript $*$ refers to a different independent turbulent experiment, different in Reynolds number and/or experimental set-up. The superscript $\leftarrow$ denotes the inverse function.

Figure 3 shows the estimated time change $\psi$ for a number of independent turbulent experiments. For a wide range of time lags, $\psi$ is essentially affine in a first approximation. The degree of non-affinity increases with increasing difference of the Reynolds numbers.

Remark 5. The collapse of the densities of velocity increments implies that the variances $\operatorname{Var}\left\{\Delta \tilde{u}_{t}\right\}$ and $\operatorname{Var}\left\{\Delta \tilde{u}_{t}^{*}\right\}$ at the corresponding time scales are the same. Denoting the variances by $c_{2}(t)=\operatorname{Var}\left\{\Delta \tilde{u}_{t}\right\}$ and $c_{2}^{*}(t)=\operatorname{Var}\left\{\Delta \tilde{u}_{t}^{*}\right\}$, the time change $\psi$ can, alternatively be expressed as (c.f. (14))

$$
\psi(t)=\overleftarrow{c}_{2}^{*}\left(c_{2}(t)\right)
$$

Remark 6. It is important to note that the quality of the collapse of the densities of velocity increments does not depend on the degree of non-affinity of the time change $\psi$. Velocity increments of widely different experiments collapse for all amplitudes at time lags at which they have the same variance [BNSch06b; BNSchS06].

\subsection{Theoretical considerations}

The empirically observed approximate affinity of the time change $\psi$ for a range of intermediate time scales can be motivated theoretically for turbulent experiments where a clear Kolmogorov scaling is observed. Such a Kolmogorov scaling is expected for large Reynolds numbers and for a certain range of scales, called the inertial range [KOL41]. In the limit of very large Reynolds numbers, the variance of velocity increments is expected to show a scaling behaviour of the form

$$
c_{2}(t)=a t^{2 / 3}
$$

where $a$ is a flow dependent constant and the time scale $t$ is within the inertial range. (In practice, one defines the inertial range as the range of time scales for which (18) holds.) For such flows the expected time change is affine (within the inertial range).

For small and moderate Reynolds numbers, the inertial range is absent or very small. For instance, the examples shown in Figure 3 do not show a clear Kolmogorov scaling for an extended range of time scales. However, the empirically estimated time change appears to be affine for a wide range of time scales. This gives the possibility to define a non-scaling counterpart of Kolmogorov scaling and an associated generalized inertial range where the variances are universal in the sense that

$$
\psi(t)=\overleftarrow{c}_{2}^{*}\left(c_{2}(t)\right)=c t+\psi_{0}
$$

where $\psi_{0}$ is a constant. A particular example are variances of the form

$$
c_{2}(t)=a\left(t+t_{0}\right)^{2 / 3},
$$

where $t_{0}$ is a constant. In view of Kolmogorov scaling, we then expect $t_{0} \rightarrow 0$ as the Reynolds number gets very large. 


\section{Modelling frameworks in finance and turbulence}

Volatility modulated Volterra processes of the form (5) have found applications in finance as well as for the modelling of the turbulent velocity field. In the turbulence context, these processes capture the main idea of the Reynolds decomposition of the velocity field into a slowly varying component (the second term in Equation (5)) and a rapidly varying component (the first term in Equation (5)) [BNSch06a; BNSch07a].

In the following subsections, we discuss the application of volatility modulated Volterra processes in finance and turbulence with emphasis on the empirical findings concerning time change and universality.

\subsection{Finance}

The basic framework for stochastic volatility modelling in finance is that of Brownian semimartingales

$$
Y_{t}=\int_{0}^{t} \sigma_{s} \mathrm{~d} B_{s}+\int_{0}^{t} a_{s} \mathrm{~d} s
$$

where $\sigma$ and $a$ are caglad processes and $B$ is Brownian motion, with $\sigma$ expressing the volatility. In general, $Y, \sigma, B$ and $a$ will be multidimensional but in the present paper we shall only consider one-dimensional processes. Importantly, whatever the process $a$, the quadratic variation of $Y$ satisfies $[Y]=\tau$ with $\tau$ given by (7).

\subsection{Turbulence}

Whereas Brownian semimartingales are 'cumulative' in nature, for free turbulence it is physically natural to model timewise velocity dynamics by stationary processes. In analogy to (19), the following framework for the latter type of dynamics has recently ([BNSch06a]) been proposed.

At time $t$ and at a fixed position in the turbulent field, the velocity of the main component of the velocity vector (i.e. the component in the mean wind direction) is specified as $V_{t}=\mu+Y_{t}$ with

$$
Y_{t}=\int_{-\infty}^{t} g(t-s) \sigma_{s} \mathrm{~d} B_{s}+\chi \int_{-\infty}^{t} q(t-s) \sigma_{s}^{2} \mathrm{~d} s .
$$

Here $B$ and $\sigma$ are as above, $\mu$ and $\chi$ are constants and $g$ and $q$ are nonnegative real functions on $(0, \infty)$ satisfying $g(0+)>0, q(0+)>0$,

$$
\|g\|^{2}=\int_{0}^{\infty} g^{2}(t) \mathrm{d} t=1
$$

and

$$
\int_{0}^{\infty} q(t) \mathrm{d} t=1
$$

Furthermore, $g$ and $q$ are assumed to be sufficiently regular to make the integrals in (20) exist, and we require that the derivative $\dot{g}$ of $g$ is square integrable.

Under these conditions, the stationary process $Y$ is a semimartingale and we have

$$
[Y]=g^{2}(0+) \tau
$$

where $\tau$ is given by (7). 
Remark 7 (Ambit processes). The model type (20) is a one-dimensional limit of a spatio-temporal modelling framework introduced under the name of Ambit processes in [BNSch0\%a]. In that more general context, the role of the Brownian motion is taken over by a Gaussian white noise field (or Brownian sheet) and the volatility is expressed as a random field, which may, for instance, be generated from a Lévy basis as in [BNSch04]. The paper [BNSch07a] gives a first discussion of the theoretical properties of such processes and describes some applications to turbulence and cancer growth.

\section{Increment processes}

Both type of processes (19) and (20) have stationary increments. In the latter case, letting $X_{t}=Y_{t}-Y_{0}$ we have

$$
\begin{aligned}
X_{t}= & \int_{-\infty}^{t}\left\{g(t-s)-1_{(-\infty, 0)}(s) g(-s)\right\} \sigma_{s} \mathrm{~d} B_{s} \\
& +\chi \int_{-\infty}^{t}\left\{q(t-s)-1_{(-\infty, 0)}(s) q(-s)\right\} \sigma_{s}^{2} \mathrm{~d} s
\end{aligned}
$$

which we also write, on VMVP form, as

$$
X_{t}=\int_{-\infty}^{t} j(t, s) \sigma_{s} \mathrm{~d} B_{s}+\chi \int_{-\infty}^{t} k(t, s) \sigma_{s}^{2} \mathrm{~d} s
$$

where

$$
\begin{aligned}
& j(t, s)=g(t-s)-1_{(-\infty, 0)}(s) g(-s) \\
& k(t, s)=q(t-s)-1_{(-\infty, 0)}(s) q(-s) .
\end{aligned}
$$

Suppose now that $B$ and $\sigma$ are independent. Clearly, $X \mid \sigma$ is then a Gaussian process with

$$
\begin{aligned}
\mathrm{E}\left\{X_{t} \mid \sigma\right\} & =\chi \int_{-\infty}^{t} k(t, s) \sigma_{s}^{2} \mathrm{~d} s \\
\operatorname{Var}\left\{X_{t} \mid \sigma\right\} & =\int_{-\infty}^{t} j^{2}(t, s) \sigma_{s}^{2} \mathrm{~d} s
\end{aligned}
$$

and, for $0 \leq s \leq t$,

$$
\operatorname{Cov}\left\{X_{s} X_{t} \mid \sigma\right\}=\int_{-\infty}^{s} j(s, u) j(t, u) \sigma_{u}^{2} \mathrm{~d} u
$$

We proceed to discuss the conditional law of $X$ given $\sigma$ and its limit behaviour for $t \rightarrow 0$ and $t \rightarrow \infty$.

Considering first the conditional mean we note that

$$
\begin{aligned}
\int_{-\infty}^{t} k(t, s) \sigma_{s}^{2} \mathrm{~d} s & =\int_{0}^{t} q(t-s) \sigma_{s}^{2} \mathrm{~d} s+\int_{-\infty}^{0}\{q(t-s)-q(-s)\} \sigma_{s}^{2} \mathrm{~d} s \\
& =\int_{0}^{t} q(s) \sigma_{t-s}^{2} \mathrm{~d} s+\int_{-\infty}^{0}\{q(t-s)-q(-s)\} \sigma_{s}^{2} \mathrm{~d} s .
\end{aligned}
$$


From this we find that

$$
\int_{-\infty}^{t} k(t, s) \sigma_{s}^{2} \mathrm{~d} s \sim q(0+) \sigma_{0}^{2} t+t \int_{0}^{\infty} q^{\prime}(s) \sigma_{-s}^{2} \mathrm{~d} s \quad \text { as } \quad t \downarrow 0
$$

and, under a mild mixing condition on $\sigma$, that

$$
\int_{-\infty}^{t} k(t, s) \sigma_{s}^{2} \mathrm{~d} s \sim K-K^{\prime} \quad \text { as } \quad t \rightarrow \infty
$$

where $K$ and $K^{\prime}$ are independent and identically distributed with

$$
K^{\prime}=\int_{0}^{\infty} q(s) \sigma_{-s}^{2} \mathrm{~d} s
$$

Similarly, for the conditional variance we have

$$
\begin{aligned}
\int_{-\infty}^{t} j^{2}(t, s) \sigma_{s}^{2} \mathrm{~d} s & =\int_{0}^{t} g^{2}(t-s) \sigma_{s}^{2} \mathrm{~d} s+\int_{-\infty}^{0}\{g(t-s)-g(-s)\}^{2} \sigma_{s}^{2} \mathrm{~d} s \\
& =\int_{0}^{t} g^{2}(s) \sigma_{t-s}^{2} \mathrm{~d} s+\int_{-\infty}^{0}\{g(t-s)-g(-s)\}^{2} \sigma_{s}^{2} \mathrm{~d} s
\end{aligned}
$$

Hence

$$
\int_{-\infty}^{t} j^{2}(t, s) \sigma_{s}^{2} \mathrm{~d} s \sim g^{2}(0+) \sigma_{0}^{2} t \quad \text { as } \quad t \downarrow 0
$$

while

$$
\int_{-\infty}^{t} j^{2}(t, s) \sigma_{s}^{2} \mathrm{~d} s \sim G+G^{\prime} \quad \text { as } \quad t \rightarrow \infty
$$

with

$$
G^{\prime}=\int_{0}^{\infty} g^{2}(s) \sigma_{s}^{2} \mathrm{~d} s
$$

and $G$ and $G^{\prime}$ independent and identical in law.

All in all we therefore have, conditionally on the volatility process $\sigma$,

$$
\frac{X_{t}}{g(0+) \sqrt{t}} \sim N\left(0, \sigma_{0}^{2}\right) \quad \text { as } \quad t \downarrow 0
$$

while

$$
X_{t} \sim N\left(\chi\left(K-K^{\prime}\right), G+G^{\prime}\right) \quad \text { as } \quad t \rightarrow \infty .
$$

In particular, the law of the increment process $X_{t}$ will not be normal in the large time scale limit unless the volatility $\sigma$ is constant. In the case of $\sigma_{t}^{2}$ following an inverse Gaussian law we get for the small time scale limit of $X_{t}$ a Normal inverse Gaussian distribution. It has been shown in [BNSch06a] that for an inverse Gaussian volatility process, the increment process is well fitted by a Normal inverse Gaussian law for all time scales. Moreover, the resulting increment process also reproduces the experimentally observed statistics of the Kolmogorov variable [Kol62]. 


\section{Time change in finance and turbulence}

The specification, in Section 6, of the modelling frameworks in finance and turbulence as specific types of VMVP now allows to discuss the idea of a time change in more detail.

\subsection{Finance}

Of particular interest are cases where the process $a$ in (19) is of the form $a=\beta \sigma^{2}$ for some constant $\beta$, i.e.

$$
Y_{t}=\int_{0}^{t} \sigma_{s} \mathrm{~d} B_{s}+\beta \tau_{t}
$$

For suitable choice of $\sigma$ this type of process is generally capable of modelling the basic dynamics of stock prices and foreign exchange rates while, at the same time, being analytically tractable. More specifically, this is the case when $\sigma^{2}$ is of supOU type with $\sigma_{t}^{2}$ following the inverse Gaussian law; see for instance [BNS01b], [BNS08].

Under the specification (23) $Y$ may, by the Dambis-Dubins-Schwartz Theorem, be rewritten as

$$
Y_{t}=B_{\tau_{t}}^{\prime}+\chi \tau_{t}
$$

where $B^{\prime}$ is a Brownian motion, which is a functional of $Y$ itself. Equivalently,

$$
Y_{\theta_{t}}=B_{t}^{\prime}+\chi t
$$

In the finance context, $\theta_{t}$ is thought of as 'operational' or 'business' time. This time is, in principle, known from the quadratic variation process $[Y]$, and that in turn can be estimated by the realised quadratic variation

$$
\left[Y_{\delta}\right]_{t}=\sum_{j=1}^{\lfloor t / \delta\rfloor}\left(Y_{j \delta}-Y_{(j-1) \delta}\right)^{2}
$$

which satisfies

$$
\left[Y_{\delta}\right] \stackrel{p}{\rightarrow}[Y]
$$

for $t \rightarrow \infty$. Equation (24) is interpreted as saying that under (23), log price returns are Gaussian when recorded in operational time. At least as a first approximation this is close to reality, see for instance [ABDE01]. Recent, more refined, empirical analysis takes the possibilities of jumps and of microstructure noise, which are not covered by (23), into account; see [ABFN06].

\subsection{Turbulence}

In this case, i.e. (20), formula (10) takes the form

$$
Y_{\theta_{t}}=\int_{-\infty}^{t} g\left(\theta_{t}-\theta_{s}\right) \mathrm{d} B_{s}+\chi \int_{-\infty}^{t} q\left(\theta_{t}-\theta_{s}\right) \mathrm{d} s .
$$

Furthermore, (11) specialises to

$$
Y_{c t+c_{0}}=\int_{-\infty}^{t} g_{c}(t-u) \sigma_{c u+c_{0}} \mathrm{~d} B_{u}^{\prime}+\chi \int_{-\infty}^{t} q_{c}(t-u) \sigma_{c u+c_{0}}^{2} \mathrm{~d} u
$$


where

$$
g_{c}(t)=\sqrt{c} g(c t) \quad \text { and } \quad q_{c}(t)=c q(c t) .
$$

\section{Universality: Modelling}

Let $V=\left\{V_{t}\right\}_{t \in \mathbb{R}}$ denote the time-wise behaviour of the mean component of the velocity vector at a fixed position in an arbitrary free turbulent field and let $U=$ $\left\{U_{t}\right\}_{t \in \mathbb{R}}$ denote the time-wise behaviour of the increment process of $V$.

\subsection{Primitive Universality Model}

We propose to consider the following as a theoretical model for the empirically observed approximate affinity of the intrinsic time change $\psi$ that results in the collapse of the densities of turbulent velocity increments.

Primitive Universality Model (PUM) Except for a change of location, scale and affine time change $T_{t}=c t+c_{0}$ (with $c>0$ and $c_{0}$ is a constant), $V$ is equivalent in law to a process $Y$ of the form

$$
Y_{t}=\int_{-\infty}^{t} g(t-s) \sigma_{s} \mathrm{~d} B_{s}+\chi \int_{-\infty}^{t} q(t-s) \sigma_{s}^{2} \mathrm{~d} s
$$

with $g, q, \sigma$ and $\chi$ as specified in connection to formula (20) and with these four quantities being universal, i.e. the same for all processes of the type $V$.

Remark 8. In this framework, once (20) is specified, an arbitrary process $V$ is characterised by $\eta=\mathrm{E}\left\{V_{0}\right\}, \omega^{2}=\operatorname{Var}\left\{Y_{0}\right\}$ and the time change constants $c$ and $c_{0}$.

\subsection{Refined Universality Model}

The empirically observed deviations from affinity of the time change $\psi$ at small and large time lags are inconsistent with the stationarity of $V$ in free turbulence. To account for this non-affine behaviour, we propose a refined universality model.

Refined Universality Model (RUM) Except for a change of scale and deterministic time change $T, U$ is equivalent in law to a process $X$ of the form

$$
X_{t}=\int_{-\infty}^{t} j(t, s) \sigma_{s} \mathrm{~d} B_{s}+\chi \int_{-\infty}^{t} k(t, s) \sigma_{s}^{2} \mathrm{~d} s
$$

with $j, k, \sigma$ and $\chi$ as specified in connection to formulae (20) and (22) and with these four quantities being universal, i.e. the same for all increment processes $U$.

Remark 9. In this framework, once (25) is specified, an arbitrary increment process $U$ is characterised by $\omega^{2}=\frac{1}{2} \lim _{t \rightarrow \infty} \operatorname{Var}\left\{Y_{t}\right\}$ and the time change $T$.

Remark 10. The empirical findings reported here only concern the collapse of the marginal distributions of velocity increments after applying a deterministic time change. The refined universality model goes beyond this equivalence in distribution as it states an equivalence in law of the processes. 


\section{Concluding remarks}

In this paper, we presented a review of some recent modelling of turbulent velocities and financial price processes and associated questions of time change. As a preliminary hypothesis, we proposed the existence of an affine time change in terms of which the velocity process is universal in law, except for change of location and scale.

The subsequent empirical findings about the non-affinity of the deterministic time change at very small and very large time scales led to us to propose the existence of a refined universality model for turbulent velocity increments and related to that an intrinsic deterministic time change, capturing the individual characteristics of each turbulent experiment. It is important to note that the empirical verification of the collapse of the densities of the time changed velocity increments is in fact independent of any model specification. Without model specification, the refined universality model can be stated as the equivalence of the law of $U$, except for change of location and scale and the time change.

A natural extension of the empirical results reported here concerns the dynamical aspect of the refined universality model, i.e. whether the empirically observed equivalence in one-dimensional marginal distribution can indeed be extended to an equivalence of the processes.

A first empirical result to clarify this point shows that the conditional distributions $p\left(\Delta \tilde{u}_{t}-\Delta \tilde{u}_{s} \mid \Delta \tilde{u}_{s}\right)$ and $p\left(\Delta \tilde{u}_{\psi(t)}^{*}-\Delta \tilde{u}_{\psi(s)}^{*} \mid \Delta \tilde{u}_{\psi(s)}^{*}\right)$ collapse after an appropriate change of scale, for $c_{2}\left(\Delta \tilde{u}_{s}\right)=c_{2}\left(\Delta \tilde{u}_{\psi(s)}^{*}\right)$ and for a range of time lags at which the time change $\psi$ is essentially affine. For time lags at which the time change $\psi$ is essentially non-affine, i.e. at very small and very large time scales, the conditional densities do not collapse; however, they are only shifted by the conditional means $\mathrm{E}\left\{\Delta \tilde{u}_{t}-\Delta \tilde{u}_{s} \mid \Delta \tilde{u}_{s}\right\}$ and $\mathrm{E}\left\{\Delta \tilde{u}_{\psi(t)}^{*}-\Delta \tilde{u}_{\psi(s)}^{*} \mid \Delta \tilde{u}_{\psi(s)}^{*}\right\}$. A further clarification of this point is outside the scope of the present paper, but will be discussed in an upcoming publication.

The definition of volatility modulated Volterra processes as given by (5) is readily generalised to the multivariate setting, with $B$ being $d$-dimensional Brownian motion and $\sigma$ being a matrix process. It is further of interest to consider cases where processes expressing possible jumps or noise in the dynamics are added.

A central issue in these settings is how to draw inference on the volatility process $\sigma$. In cases where the processes are semimartingales, the theory of multipower variations (see [BNGJPS07], [BNGJS06] and references given there) provides effective tools for this.

However, VMVP processes are generally not of semimartingale type and the question of how to proceed then is largely unsolved and poses mathematically challenging problems. Some of these problems are presently under study in joint work with José-Manuel Corcuera, Mark Podolski and Neil Shephard.

\section{A The Normal inverse Gaussian Law}

The class of NIG distributions equals the family of possible distributions at time $t=1$ of the NIG Lévy process, which is defined as Brownian motion with drift 
subordinated by the inverse Gaussian Lévy process, i.e. the Lévy process of first passage times to constant levels of (another, independent) Brownian motion.

The normal inverse Gaussian law, with parameters $\alpha, \beta, \mu$ and $\delta$, is the distribution on the real axis $\mathbf{R}$ having probability density function

$$
\begin{aligned}
p(x ; \alpha, \beta, \mu, \delta)= & a(\alpha, \beta, \mu, \delta) q\left(\frac{x-\mu}{\delta}\right)^{-1} \\
& \times K_{1}\left\{\delta \alpha q\left(\frac{x-\mu}{\delta}\right)\right\} e^{\beta x}
\end{aligned}
$$

where $q(x)=\sqrt{1+x^{2}}$ and

$$
a(\alpha, \beta, \mu, \delta)=\pi^{-1} \alpha \exp \left\{\delta \sqrt{\alpha^{2}-\beta^{2}}-\beta \mu\right\}
$$

and where $K_{1}$ is the modified Bessel function of the third kind and index 1 . The domain of variation of the parameters is given by $\mu \in \mathbf{R}, \delta \in \mathbf{R}_{+}$, and $0 \leq|\beta|<\alpha$. The distribution is denoted by $\operatorname{NIG}(\alpha, \beta, \mu, \delta)$.

If $X$ is a random variable with distribution $\operatorname{NIG}(\alpha, \beta, \mu, \delta)$ then the cumulant generating function of $X$, i.e. $\mathrm{K}(\theta ; \alpha, \beta, \mu, \delta)=\log \mathrm{E}\left\{e^{\theta X}\right\}$, has the form

$$
\mathrm{K}(\theta ; \alpha, \beta, \mu, \delta)=\delta\left\{\sqrt{\alpha^{2}-\beta^{2}}-\sqrt{\alpha^{2}-(\beta+\theta)^{2}}\right\}+\mu \theta .
$$

It follows immediately from this that if $x_{1}, \ldots, x_{m}$ are independent normal inverse Gaussian random variables with common parameters $\alpha$ and $\beta$ but individual location-scale parameters $\mu_{i}$ and $\delta_{i}(i=1, \ldots, m)$ then $x_{+}=x_{1}+\cdots+x_{m}$ is again distributed according to a normal inverse Gaussian law, with parameters $\left(\alpha, \beta, \mu_{+}, \delta_{+}\right)$.

Furthermore, the first four cumulants of $\operatorname{NIG}(\alpha, \beta, \mu, \delta)$, obtained by differentiation of (28), are found to be

$$
\kappa_{1}=\mu+\frac{\delta \rho}{\sqrt{1-\rho^{2}}}, \quad \kappa_{2}=\frac{\delta}{\alpha\left(1-\rho^{2}\right)^{3 / 2}}
$$

and

$$
\kappa_{3}=\frac{3 \delta \rho}{\alpha^{2}\left(1-\rho^{2}\right)^{5 / 2}}, \quad \kappa_{4}=\frac{3 \delta\left(1+4 \rho^{2}\right)}{\alpha^{3}\left(1-\rho^{2}\right)^{7 / 2}},
$$

where $\rho=\beta / \alpha$. Hence, the standardised third and fourth cumulants are

$$
\begin{aligned}
\bar{\kappa}_{3} & =\frac{\kappa_{3}}{\kappa_{2}^{3 / 2}}=3 \frac{\rho}{\left\{\delta \alpha\left(1-\rho^{2}\right)^{1 / 2}\right\}^{1 / 2}} \\
\bar{\kappa}_{4} & =\frac{\kappa_{4}}{\kappa_{2}^{2}}=3 \frac{1+4 \rho^{2}}{\delta \alpha\left(1-\rho^{2}\right)^{1 / 2}} .
\end{aligned}
$$

We note that the NIG distribution (26) has semiheavy tails; specifically,

$$
p(x ; \alpha, \beta, \mu, \delta) \sim \text { const. }|x|^{-3 / 2} \exp (-\alpha|x|+\beta x), x \rightarrow \pm \infty
$$

as follows from the asymptotic relation

$$
K_{\nu}(x) \sim \sqrt{2 / \pi} x^{-1 / 2} e^{-x} \text { as } x \rightarrow \infty .
$$


It is often of interest to consider alternative parametrisations of the normal inverse Gaussian laws. In particular, letting $\bar{\alpha}=\delta \alpha$ and $\bar{\beta}=\delta \beta$, we have that $\bar{\alpha}$ and $\bar{\beta}$ are invariant under location-scale changes. Note that $\rho=\bar{\beta} / \bar{\alpha}$.

NIG shape triangle For some purposes it is useful, instead of the classical skewness and kurtosis quantities (31), to work with the alternative asymmetry and steepness parameters $\chi$ and $\xi$ defined by

$$
\chi=\rho \xi
$$

and

$$
\xi=(1+\bar{\gamma})^{-1 / 2}
$$

where $\rho=\beta / \alpha=\bar{\beta} / \bar{\alpha}$ and $\bar{\gamma}=\delta \gamma=\delta \sqrt{\alpha^{2}-\beta^{2}}$. Like $\bar{\kappa}_{3}$ and $\bar{\kappa}_{4}$, these parameters are invariant under location-scale changes and the domain of variation for $(\chi, \xi)$ is the normal inverse Gaussian shape triangle

$$
\{(\chi, \xi):-1<\chi<1,0<\xi<1\}
$$

The distributions with $\chi=0$ are symmetric, and the normal and Cauchy laws occur as limiting cases for $(\chi, \xi)$ near to $(0,0)$ and $(0,1)$, respectively. Figure 4 gives an impression of the shape of the NIG distributions for various values of $(\chi, \xi)$.

Note in this connection that $\bar{\kappa}_{3}$ and $\bar{\kappa}_{4}$ may be reexpressed as

$$
\bar{\kappa}_{3}=3 \bar{\gamma}^{-1} \frac{\rho}{\left\{\left(1+\rho^{2}\right)\left(1-\rho^{2}\right)^{1 / 2}\right\}^{1 / 2}}
$$

and

$$
\bar{\kappa}_{4}=3 \bar{\gamma}^{-1} \frac{1+4 \rho^{2}}{\left(1-\rho^{4}\right)^{1 / 2}}
$$

from which it follows that for small $\rho$ we have approximately $\xi \doteq\left(1+3 / \bar{\kappa}_{4}\right)^{-1 / 2}$ and $\bar{\kappa}_{3} \doteq \rho \bar{\kappa}_{4}$ (compare to (34)); Thus the roles of $\chi$ and $\xi$ are rather similar to those of the classical quantities $\bar{\kappa}_{3}$ and $\bar{\kappa}_{4}$.

A systematic study of the class of normal inverse Gaussian distributions, and of associated stochastic processes, was begun in [BN95; BN97; BN98a; BN98b; BN98c]. Further theoretical developments and applications are discussed in [RYD97; RYD99; PR99; EB00; RAI00; BNS01a; BNS01b; BNS02; BNP01; BNL01; ASR01; CONT04; FOR02; MC05]. As discussed in the papers cited and in references given there, the class of NIG distributions and processes have been found to provide accurate modelling of a great variety of empirical findings in the physical sciences and in financial econometrics. (The wider class of generalised hyperbolic distributions, introduced in [BN77], provides additional possibilities for realistic modelling of dynamical processes, see references in the papers cited above.) 


\section{References}

[ABDE01] Andersen, T.G., Bollerslev, T., Diebold, F.X. and Ebens, H. (2001): The distribution of realized stock return volatility. J. Fin. Econometrics $\mathbf{6 1}, 43-76$.

[ABFN06] Andersen, T.G., Bollerslev, T., Frederiksen, P.H. and Nielsen, M.Ø. (2006): Continuous-time models, realized volatilities, and testable distributional implications for daily stock returns. Unpublished paper.

[ASR01] Asmussen, S. and Rosinski, J. (2001): Approximation of small jumps of Lévy processes with a view towards simulation. J. Appl. Probab. 38, 482-493.

[BN77] Barndorff-Nielsen, O.E. (1977): Exponentially decreasing distributions for the logarithm of particle size. Proc. R. Soc. London A 353, 401419.

[BN95] Barndorff-Nielsen, O.E. (1995): Normal inverse Gaussian processes and the modelling of stock returns. Research Report 300, Dept. Theor. Statistics, Aarhus University.

[BN97] Barndorff-Nielsen, O.E. (1997): Normal inverse Gaussian distributions and stochastic volatility modelling. Scand. J. Statist. 24, 1-14.

[BN98a] Barndorff-Nielsen, O.E. (1998a): Probability and statistics: selfdecomposability, finance and turbulence. In Acccardi, L. and Heyde, C.C. (Eds.): Probability Towards 2000. Proceedings of a Symposium held 2-5 October 1995 at Columbia University. New York: SpringerVerlag. Pp. 47-57.

[BN98b] Barndorff-Nielsen, O.E. (1998b): Processes of normal inverse Gaussian type. Finance and Stochastics 2, 41-68.

[BN98c] Barndorff-Nielsen, O.E. (1998c): Superposition of Ornstein-Uhlenbeck type processes. Theory Prob. Its Appl. 45, 175-194.

[BNBSch04] Barndorff-Nielsen, O.E., Blæsild, P. and Schmiegel, J. (2004): A parsimonious and universal description of turbulent velocity increments. Eur. Phys. J. B 41, 345-363.

[BNGJPS07] Barndorff-Nielsen, O.E., Graversen, S.E., Jacod, J., Podolskij, M. and Shephard, N. (2006): A central limit theorem for realised power and bipower variations of continuous semimartingales. In Yu. Kabanov, R. Liptser and J. Stoyanov (Eds.): From Stochastic Calculus to Mathematical Finance. Festschrift in Honour of A.N. Shiryaev. Heidelberg: Springer. Pp. 33-68.

[BNGJS06] Barndorff-Nielsen, O.E., Graversen, S.E., Jacod, J. and Shephard, N. (2006): Limit theorems for bipower variation in financial econometrics. Econometric Theory 22, 677-719. 
[BNL01] Barndorff-Nielsen, O.E. and Levendorskiŭ, S.Z. (2001): Feller processes of normal inverse Gaussian type. Quantitative Finance 1, 318-331.

[BNP01] Barndorff-Nielsen, O.E. and Prause, K. (2001): Apparent scaling. Finance and Stochastics. 5, 103-113.

[BNSch04] Barndorff-Nielsen, O.E. and Schmiegel, J. (2004): Lévy-based tempospatial modelling; with applications to turbulence. Uspekhi Mat. NAUK 59, 65-91.

[BNSch06a] Barndorff-Nielsen, O.E. and Schmiegel, J. (2006a): A stochastic differential equation framework for the timewise dynamics of turbulent velocities. Theory of Probability and its Applications. (To appear.)

[BNSch06b] Barndorff-Nielsen, O.E. and Schmiegel, J. (2006b): Time change and universality in turbulence. (Submitted.)

[BNSch07a] Barndorff-Nielsen, O.E. and Schmiegel, J. (2007a): Ambit processes; with applications to turbulence and cancer growth. Proceedings of the 2005 Abel Symposium on Stochastic Analysis and Applications. Heidelberg: Springer. (To appear.)

[BNSch07b] Barndorff-Nielsen, O.E. and Schmiegel, J. (2007b): Change of time and universal laws in Turbulence. (Submitted.)

[BNSchS06] Barndorff-Nielsen, O.E., Schmiegel, J. and Shephard, N. (2006): Time change and universality in turbulence and finance. (Submitted.)

[BNSchS07] Barndorff-Nielsen, O.E., Schmiegel, J. and Shephard, N. (2007): QV, $\mathrm{BV}$ and VR under stationary Gaussian processes. (In preparation.)

[BNS01a] Barndorff-Nielsen, O.E. and Shephard, N. (2001a): Modelling by Lévy processes for financial econometrics. In O.E. Barndorff-Nielsen, T. Mikosch and S. Resnick (Eds.): Lévy Processes - Theory and Applications. Boston: Birkhäuser. Pp. 283-318.

[BNS01b] Barndorff-Nielsen, O.E. and Shephard, N. (2001): Non-Gaussian Ornstein-Uhlenbeck-based models and some of their uses in financial economics (with Discussion). J. R. Statist. Soc. B 63, 167-241.

[BNS02] Barndorff-Nielsen, O.E. and Shephard, N. (2002): Integrated OU processes and non-Gaussian OU-based stochastic volatility. Scand. J. Statist. 30, 277-295.

[BNS04] Barndorff-Nielsen, O.E. and Shephard, N. (2004): Power and bipower variation with stochastic volatility and jumps (with Discussion). $J$. Fin. Econometrics 2, 1-48.

[BNS08] Barndorff-Nielsen, O.E. and Shephard, N. (2008): Financial Volatility in Continous Time. Cambridge University Press. (To appear.) 
[BNShi08] Barndorff-Nielsen, O.E. and Shiryaev, A.N. (2008): Change of Time and Change of Measure. Singapore: World Scientific. (To appear.)

[BeMar07] Bender, C. and Marquardt, T. (2007): Stochastic calculus for convoluted Lévy processes. (Unpublished manuscript.)

[Cas90] Castaing, B., Gagne, Y. and Hopfinger, E.J. (1990): Velocity probability density functions of high Reynolds number turbulence. Physica D 46, 177-200.

[CONT04] Cont, R. and Tankov, P. (2004): Financial Modelling With Jump Processes. Chapman \& Hall/CRC, London.

[Dec05] Decreusefond, L. (2005): Stochastic integration with respect to Volterra processes. Ann. I. H. Poincaré PR41, 123-149.

[DecSa06] Decreusefond, L. and Savy, N. (2006): Anticipative calculus with respect to filtered Poisson processes. Ann. I. H. Poincaré PR42, 343372.

[EB00] Eberlein, E. (2000): Application of generalized hyperbolic Lévy motion to finance. In O.E. Barndorff-Nielsen, T. Mikosch and S. Resnick (Eds.): Lévy Processes - Theory and Applications. Boston: Birkhäuser. Pp. 319-336.

[Els96] Elsner, J.W. and Elsner, W. (1996): On the measurement of turbulence energy dissipation. Meas. Sci. Technol. 7, 1334-1348.

[FOR02] Forsberg, L. (2002): On the Normal Inverse Gaussian distribution in Modelling Volatility in the Financial Markets. Acta Universitatis Upsaliemsis, Studia Statistica Upsaliensia 5, Uppsala.

[Gha96] Ghashgaie, S., Breymann, W., Peinke, J., Talkner, P. and Dodge, Y. (1996): Turbulent cascades in foreign exchange markets. Nature $\mathbf{3 8 1}$, $767-770$

[Hu03] Hult, H. (2003): Approximating some Volterra type stochastic intergrals with applications to parameter estimation. Stoch. Proc. Appl. 105, 1-32.

[KOL41] Kolmogorov, A.N. (1941): Dissipation of energy in locally isotropic turbulence. Dokl. Akad. Nauk. SSSR 32, 16-18.

[Kol62] Kolmogorov, A.N. (1962): A refinement of previous hypotheses concerning the local structure of turbulence in a viscous incompressible fluid at high Reynolds number, J. Fluid Mech 13, 82-85.

[Mar06] Marquardt, T. (2006): Fractional Lévy processes with an application to long memory moving average processes. Bernoulli 12, 1099-1126.

[MC05] McNeil, A.J., Frey, R. and Embrechts, P. (2005): Quantitative Risk Management. Princton University Press, Princeton. 
[NoVaVir99] Norros, I., Valkeila, E. and Virtamo, J. (1999): An elementary approach to a Girsanov formula and other analytic results on fractional Brownian motion. Bernoulli 5, 571-587.

[Obu62] Obukhov, A.M. (1962): Some specific features of atmospheric turbulence. J. Fluid Mech. 13, 77-81.

[Pei04] Peinke, J., Bottcher, F. and Barth, S. (2004): Anomalous statistics in turbulence, financial markets and other complex systems. Ann. Phys. (Leipzig) 13, 450-460.

[PR99] Prause, K. (1999): The Generalized Hyperbolic Model: Estimation, Financial Derivatives and Risk Measures. Dissertation. Albert-LudwigsUniversität, Freiburg i. Br.

[RAI00] Raible, S. (2000): Lévy Processes in Finance: Theory, Numerics, Empirical Facts. Dissertation. Albert-Ludwigs-Universität, Freiburg i. Br.

[RYD97] Rydberg, T.H. (1997): The normal inverse Gaussian Lévy process: simulation and approximation. Comm. Statist.: Stochastic Models 13, 887-910.

[RYD99] Rydberg, T.H. (1999): Generalized hyperbolic diffusions with applications towards finance. Math. Finance 9 , 183-201.

[SaTa94] Samorodnitsky, G. and Taqqu, M.S. (1994); Stable Non-Gaussian Random Processes. New York: Chapman and Hall.

[Shi06] Shiryaev, A.N. (2006): Kolmogorov and the turbulence. Research Report 2006-4. Thiele Centre for Applied Mathematics in Natural Science.

[Shi07] Shiryaev, A.N. (2007): On the classical, statistical and stochastic approaches to hydrodynamic turbulence. Research Report 2007-2. Thiele Centre for Applied Mathematics in Natural Science.

[Vin91] Vincent, A. and Meneguzzi, M. (1991): The spatial structure and statistical properties of homogeneous turbulence. J. Fluid Mech. 225, $1-25$. 


\begin{tabular}{lcc}
\hline varying activity & $\begin{array}{c}\text { Finance } \\
\text { volatility }\end{array}$ & $\begin{array}{c}\text { Turbulence } \\
\text { intermittency }\end{array}$ \\
\hline semiheavy tails & + & + \\
asymmetry & + & + \\
aggregational Gaussianity & + & + \\
0 autocorrelation & + & - \\
quasi long range dependence & + & + \\
scaling/selfsimilarity & {$[+]$} & {$[+]$} \\
leverage & + & - \\
operational time & + & + \\
trend & cumulative & stationary \\
jumps & + & - \\
\hline
\end{tabular}

Table 1: Stylised features of turbulence and finance. 

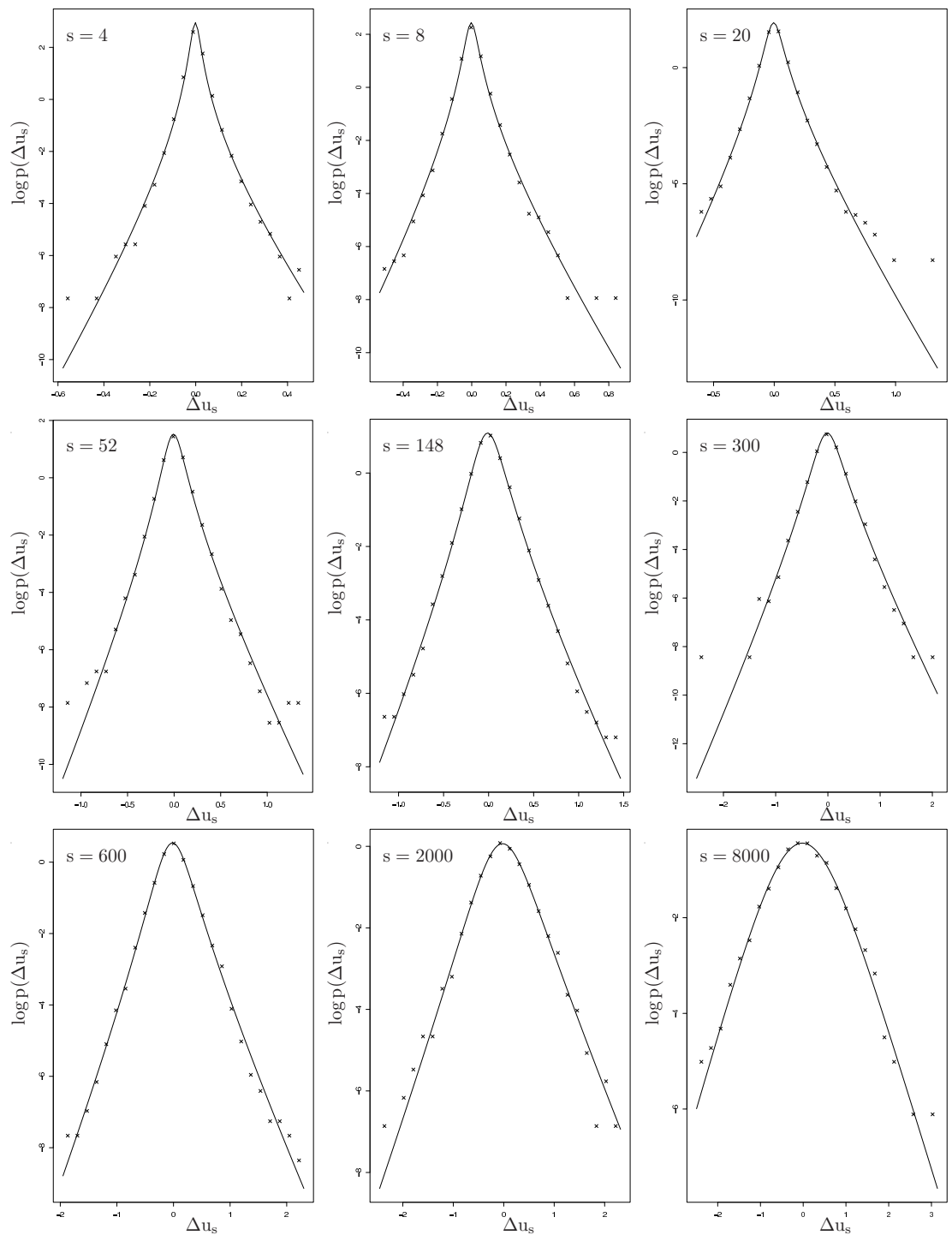

Figure 1: Approximation of the pdf of velocity increments within the class of NIG distributions (solid lines, fitting by maximum likelihood) for data from the atmospheric boundary layer (kindly provided by K.R. Sreenivasan) with $R_{\lambda}=17000$ and time scales $s=4,8,20,52,148,300,600,2000,8000$ (in units of the finest resolution). 

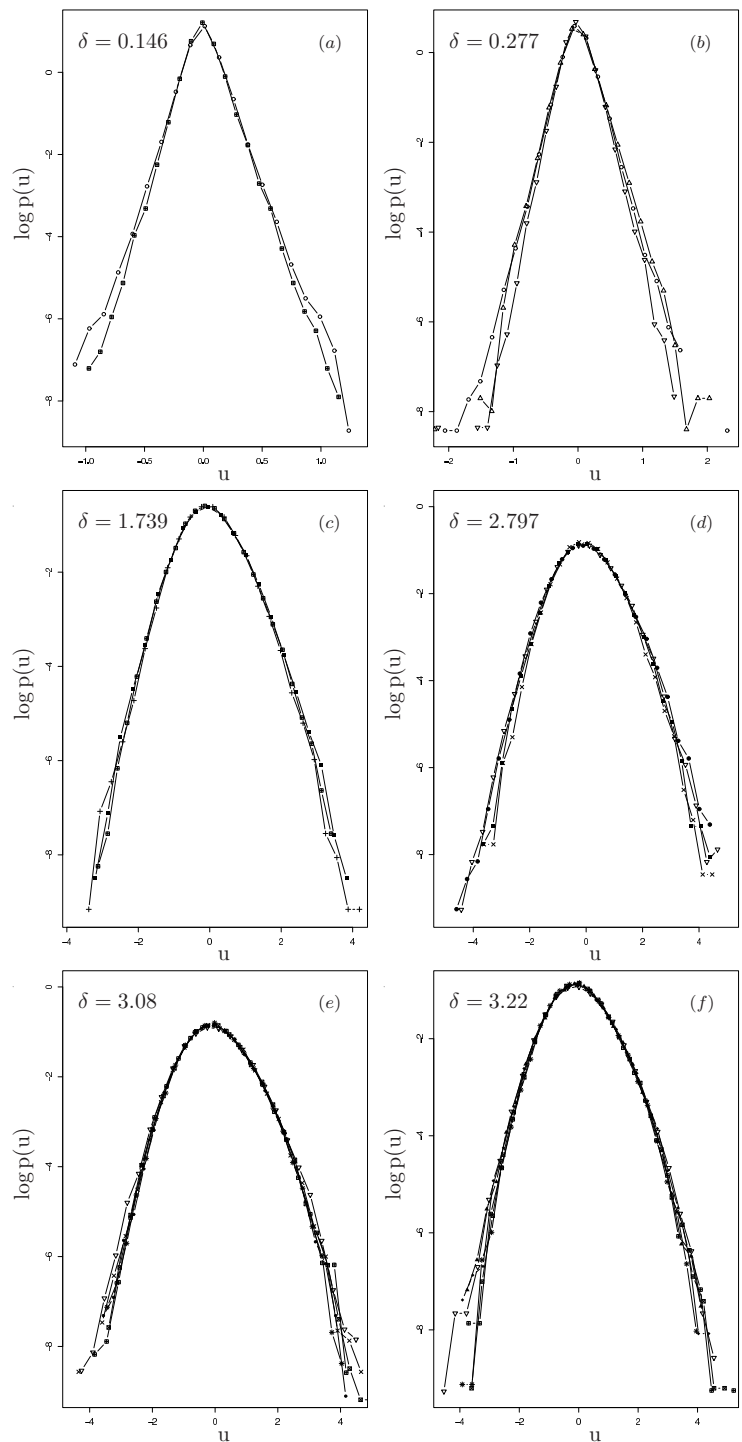

Figure 2: Collapse of the densities of velocity increments at time scale $s$ for various fixed values of the scale parameter $\delta(s)$ of the approximating NIG-distributions. The data are from the atmospheric boundary layer (data set (at) with $R_{\lambda}=17000$, kindly provided by K.R. Sreenivasan), from a free jet experiment (data set (j) with $R_{\lambda}=190$, kindly provided by J. Peinke), from a wind tunnel experiment (data set (w) with $R_{\lambda}=80$, kindly provided by B.R. Pearson) and from a gaseous helium jet flow (data sets (h85), (h124), (h208), (h283), (h352), (h703), (h885), (h929), (h985) and (h1181) with $R_{\lambda}=85,124,208,283,352,703,885,929,985,1181$, respectively, kindly provided by B. Chabaud). The corresponding values of the time scales $s$ (in units of the finest resolution of the corresponding data set) and the codes for the data sets are $(a)(s=116$, (at)) $(\circ),(s=4,(\mathrm{~h} 352))(\boxplus),(b)(s=440,(\mathrm{at}))(\circ),(s=8,(\mathrm{j}))(\triangle),(s=8,(\mathrm{~h} 929))(\nabla),(c)$ $(s=192,(\mathrm{~h} 885))(\mathbf{\square}),(s=88,(\mathrm{~h} 352))(\boxplus),(s=10,(\mathrm{w}))(+),(d)(s=380,(\mathrm{~h} 885))$ $(s=410,(\mathrm{~h} 929))(\nabla),(s=350,(\mathrm{~h} 703))(\times),(s=340,(\mathrm{~h} 985))(\bullet),(e)(s=420,(\mathrm{~h} 703))$ $(\times),(s=440,(\mathrm{~h} 929))(\nabla),(s=180,(\mathrm{~h} 352))(\boxplus),(s=270,(\mathrm{~h} 283))(\bullet),(s=108,(\mathrm{~h} 124))$ $(*),(s=56,(\mathrm{~h} 85))(\otimes),(f)(s=470,(\mathrm{~h} 929))(\nabla),(s=116,(\mathrm{~h} 124))(*),(s=60,(\mathrm{~h} 85))$ $(\bigotimes),(s=188,(\mathrm{~h} 352))(\boxplus),(s=470,(\mathrm{~h} 1181))(\mathbf{\Delta}),(s=140,(\mathrm{~h} 208))(\diamond)$. 

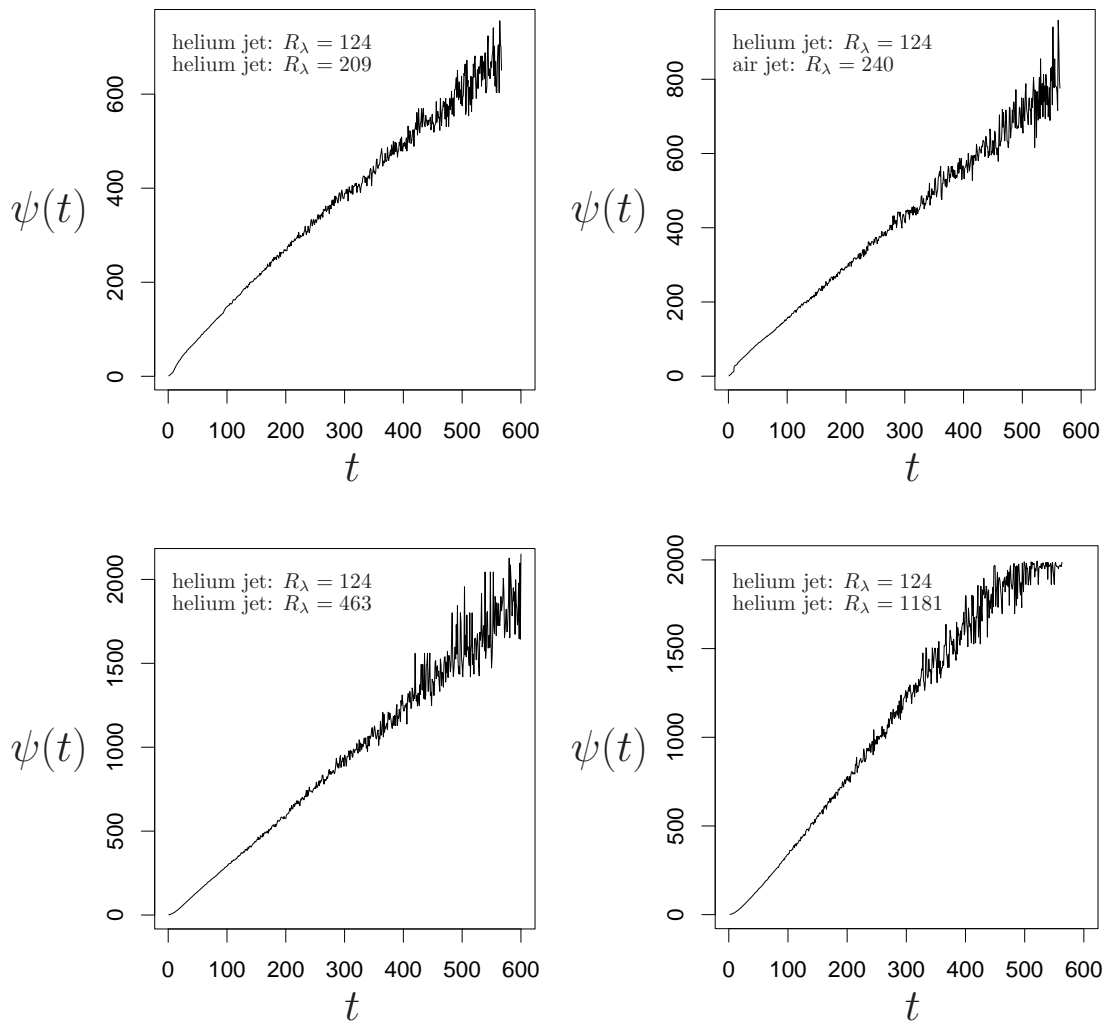

Figure 3: Estimated time change $\psi$ (in units of the finest resolution of the respective data sets) resulting in a collapse of the densities of velocity increments (see Figure 2).

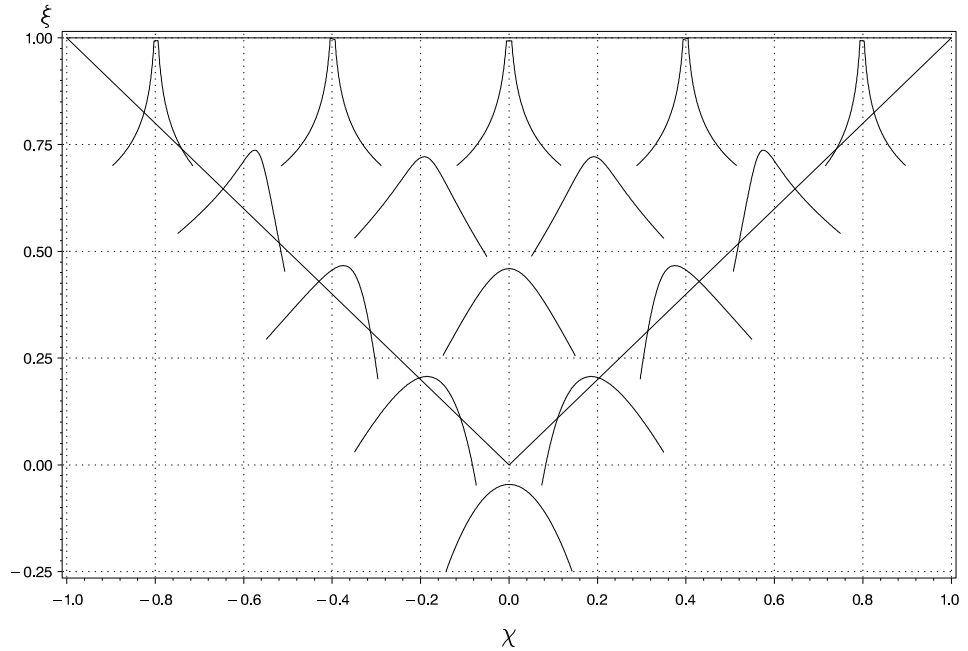

Figure 4: The shape triangle of the NIG distributions with the log density functions of the standardized distributions, i.e. with mean 0 and variance 1 , corresponding to the values $(\chi, \xi)=( \pm 0.8,0.999),( \pm 0.4,0.999),(0.0,0.999),( \pm 0.6,0.75),( \pm 0.2,0.75),( \pm 0.4,0.5)$, $(0.0,0.5),( \pm 0.2,0.25)$ and $(0.0,0.0)$. The coordinate system of the $\log$ densities is placed at the corresponding value of $(\chi, \xi)$. 